UWThPh-2005-18

OCHA-PP-255

NIIG-DP-05-2

\title{
$\mu-\tau$ antisymmetry and neutrino mass matrices
}

\author{
Walter Grimus* \\ Institut für Theoretische Physik, Universität Wien \\ Boltzmanngasse 5, A-1090 Wien, Austria \\ Satoru Kaneko ${ }^{\dagger}$ \\ Department of Physics, Ochanomizu University, Tokyo 112-8610, Japan \\ Luís Lavoura ${ }^{\ddagger}$ \\ Universidade Técnica de Lisboa and Centro de Física Teórica de Partículas \\ Instituto Superior Técnico, 1049-001 Lisboa, Portugal \\ Hideyuki Sawanaka ${ }^{\S}$ \\ Graduate School of Science and Technology, Niigata University, 950-2181 Niigata, Japan \\ Morimitsu Tanimoto \\ Department of Physics, Niigata University, 950-2181 Niigata, Japan
}

25 October 2005

\begin{abstract}
Using the seesaw mechanism and a discrete symmetry, we construct a class of models for the neutrino mass matrix where the inverse of that matrix is the sum of a $\mu-\tau$ antisymmetric background and a perturbation. We consider various possibilities for that perturbation. The simplest possible perturbations lead to fourparameter neutrino mass matrices which are unable to fit the experimental data. More complicated perturbations give rise to viable six-parameter mass matrices; we present detailed predictions of each of them.
\end{abstract}

\footnotetext{
*E-mail: walter.grimus@univie.ac.at

${ }^{\dagger}$ E-mail: satoru@phys.ocha.ac.jp

${ }_{\ddagger}^{\ddagger}$ E-mail: balio@cftp.ist.utl.pt

$\S$ E-mail: hide@muse.sc.niigata-u.ac.jp

ฯE-mail: tanimoto@muse.sc.niigata-u.ac.jp
} 


\section{Introduction}

The present experimental data on lepton mixing [1, 2] are compatible with maximal atmospheric neutrino mixing. One possible theoretical explanation (for recent reviews see [3]) for that maximal mixing, if it indeed occurs, is $\mu-\tau$ interchange symmetry [4, 5]. In the basis where the charged-lepton mass matrix is diagonal, we denote the effective light-neutrino Majorana mass matrix by $\mathcal{M}_{\nu}$. The diagonalization of $\mathcal{M}_{\nu}$ is performed as

$$
U^{T} \mathcal{M}_{\nu} U=\operatorname{diag}\left(m_{1}, m_{2}, m_{3}\right)
$$

where $m_{1,2,3}$ are the neutrino masses and $U$ is the (unitary) lepton mixing matrix. If $\mathcal{M}_{\nu}$ is $\mu-\tau$ symmetric, i.e. if $\mathcal{M}_{\nu}=M^{(\mathrm{S})}$ with

$$
M^{(\mathrm{S})}=\left(\begin{array}{ccc}
x & y & y \\
y & z & w \\
y & w & z
\end{array}\right),
$$

then atmospheric neutrino mixing is maximal, i.e. $\left|U_{\mu 3}\right|=\left|U_{\tau 3}\right|$. Indeed, the matrix (2) has six physical parameters, since only the phases of $z w^{*}$ and of $y^{2} x^{*} z^{*}$ are physical. The existence of only six physical parameters in a theory for nine observables - the three neutrino masses $m_{1,2,3}$, the three lepton mixing angles $\theta_{23,13,12}$, one Dirac phase $\delta$, and two Majorana phases $\Theta$ and $\Omega$-leads to three predictions. ${ }^{1}$ In this case the predictions are the following: $\left|U_{\mu 3}\right|=\left|U_{\tau 3}\right|\left(\theta_{23}=\pi / 4\right), U_{e 3}=0\left(\theta_{13}=0\right)$, and the Dirac phase $\delta$ is meaningless since $U$ has one vanishing matrix element.

The $\mu-\tau$ interchange symmetry, represented by the matrix

$$
T=\left(\begin{array}{lll}
1 & 0 & 0 \\
0 & 0 & 1 \\
0 & 1 & 0
\end{array}\right),
$$

is a $\mathbb{Z}_{2}$ symmetry, and as such it has two possible eigenvalues: +1 and -1 . The matrix $M^{(\mathrm{S})}$ corresponds to the eigenvalue $+1: T M^{(\mathrm{S})} T=+M^{(\mathrm{S})}$. But there is also the possibility that $\mathcal{M}_{\nu}$ is $\mu-\tau$ antisymmetric [6], i.e. that $\mathcal{M}_{\nu}=M^{(\mathrm{AS})}$, where $T M^{(\mathrm{AS})} T=-M^{(\mathrm{AS})}$ or

$$
M^{(\mathrm{AS})}=\left(\begin{array}{ccc}
0 & y & -y \\
y & z & 0 \\
-y & 0 & -z
\end{array}\right) .
$$

This possibility seems attractive since $M^{(\mathrm{AS})}$ may be diagonalized as

$$
V^{T} M^{(\mathrm{AS})} V=\operatorname{diag}(i k,-i k, 0),
$$

where $k=\sqrt{2|y|^{2}+|z|^{2}}$ and the unitary matrix $V$ is

$$
V=\frac{1}{2 k}\left(\begin{array}{ccc}
2 y^{*} & 2 y^{*} & -2 z \\
z^{*}+i k & z^{*}-i k & 2 y \\
z^{*}-i k & z^{*}+i k & 2 y
\end{array}\right) .
$$

\footnotetext{
${ }^{1}$ We defer the precise definition of the mixing angles, Dirac phase, and Majorana phases to section 3.
} 
Thus, $\mathcal{M}_{\nu}=M^{(\mathrm{AS})}$ leads to two degenerate massive neutrinos, one massless neutrino, maximal atmospheric neutrino mixing, and maximal solar neutrino mixing $\left(\left|U_{e 1}\right|=\left|U_{e 2}\right|\right.$ or $\left.\theta_{12}=\pi / 4\right)$. These predictions are not so far from reality, if the neutrino mass spectrum is inverted. However, the exact equality $\mathcal{M}_{\nu}=M^{(\mathrm{AS})}$ can be excluded, since we know that all three neutrino masses are non-degenerate and that solar neutrino mixing is large but not maximal.

In this paper we consider various possible perturbations to $\mu-\tau$ antisymmetry. More precisely, our starting point is

$$
\mathcal{M}_{\nu}^{-1}=M^{(\mathrm{AS})}+M^{(\mathrm{P})},
$$

where $M^{(\mathrm{P})}$ is a $\mu-\tau$ symmetric matrix, a "perturbation" to $M^{(\mathrm{AS})}$. Having $\mathcal{M}_{\nu}^{-1}$ instead of $\mathcal{M}_{\nu}$ in equation (7) is quite advantageous for model building; this will become evident in section 2, where we construct a class of models based on equation (7). Physically, exchanging $\mathcal{M}_{\nu}^{-1}$ with $\mathcal{M}_{\nu}$ simply corresponds to complex-conjugating $U$ and trading the neutrino masses by their inverses. In our class of models, the perturbation $M^{(\mathrm{P})}$ is mostly free, since it depends on the assumed scalar content of each model. We proceed in the following sections to analyze in detail the consequences of some perturbations. We show analytically in sections $3-5$ that the simplest possible perturbations lead to very constrained neutrino mass matrices, which contradict the experimental data. We proceed in section 6 to analyze numerically some more complicated perturbations wherein $\mathcal{M}_{\nu}$ has six physical parameters. We summarize our findings in section 7 .

\section{A class of models}

Consider a $S U(2) \times U(1)$ gauge theory with three scalar $S U(2)$ doublets $\phi_{j}(j=1,2,3)$. Besides the left-handed lepton $S U(2)$ doublets $D_{\alpha}$ and the right-handed charged-lepton $S U(2)$ singlets $\alpha_{R}(\alpha=e, \mu, \tau)$, we introduce right-handed neutrinos $\nu_{\alpha R}$. We assume the validity of the lepton-number $U(1)$ symmetries $L_{\alpha}$ for all dimension- 4 terms in the Lagrangian; dimension-3 terms are allowed to softly break the $L_{\alpha}$. We assume the existence of a symmetry $\mathbb{Z}_{2}^{(\text {aux })}$ under which $\phi_{1}, e_{R}$, and all the $\nu_{\alpha R}$ change sign, while all other fields remain invariant. The Yukawa Lagrangian of the scalar doublets ${ }^{2}$ is then

$$
\begin{aligned}
\mathcal{L}_{\mathrm{Y} \phi}= & -\left(y_{1} \bar{D}_{e} \nu_{e R}+y_{2} \bar{D}_{\mu} \nu_{\mu R}+y_{2}^{\prime} \bar{D}_{\tau} \nu_{\tau R}\right) i \tau_{2} \phi_{1}^{*}-y_{3} \bar{D}_{e} e_{R} \phi_{1} \\
& -\left(y_{4} \bar{D}_{\mu} \mu_{R}+y_{4}^{\prime} \bar{D}_{\tau} \tau_{R}\right) \phi_{2}-\left(y_{5} \bar{D}_{\mu} \mu_{R}+y_{5}^{\prime} \bar{D}_{\tau} \tau_{R}\right) \phi_{3}+\text { H.c. }
\end{aligned}
$$

In the $\mu-\tau$ symmetric model built by two of us a few years ago [7, a $\mu-\tau$ interchange symmetry $\mathbb{Z}_{2}^{(\mathrm{tr})}$ was introduced, under which $D_{\mu} \leftrightarrow D_{\tau}, \mu_{R} \leftrightarrow \tau_{R}, \nu_{\mu R} \leftrightarrow \nu_{\tau R}$, and $\phi_{3} \rightarrow-\phi_{3}$, such that after two applications of $\mathbb{Z}_{2}^{(\mathrm{tr})}$ all fields transform into themselves. Instead, in the present model we employ a symmetry $\mathbb{Z}_{4}$ under which

$$
\begin{aligned}
& D_{e} \rightarrow i D_{e}, \quad D_{\mu} \rightarrow i D_{\tau}, \quad D_{\tau} \rightarrow i D_{\mu}, \\
& e_{R} \rightarrow i e_{R}, \quad \mu_{R} \rightarrow i \tau_{R}, \quad \tau_{R} \rightarrow i \mu_{R}, \\
& \nu_{e R} \rightarrow i \nu_{e R}, \quad \nu_{\mu R} \rightarrow i \nu_{\tau R}, \quad \nu_{\tau R} \rightarrow i \nu_{\mu R}, \\
& \phi_{3} \rightarrow-\phi_{3} \text {. }
\end{aligned}
$$

\footnotetext{
${ }^{2}$ We shall soon introduce some scalar $S U(2)$ singlets into the theory, which will have further Yukawa interactions.
} 
Notice that all lepton fields change sign after two applications of this $\mathbb{Z}_{4}$. Just as $\mathbb{Z}_{2}^{(\operatorname{tr})}$ in [7], the present $\mathbb{Z}_{4}$ enforces $y_{2}^{\prime}=y_{2}, y_{4}^{\prime}=y_{4}$, and $y_{5}^{\prime}=-y_{5}$. The neutrino Dirac mass matrix $M_{D}$ and the charged-lepton mass matrix $M_{\ell}$ are then

$$
\begin{aligned}
M_{D} & =\operatorname{diag}(a, b, b), \\
M_{\ell} & =\operatorname{diag}\left(e^{i \varphi_{e}} m_{e}, e^{i \varphi_{\mu}} m_{\mu}, e^{i \varphi_{\tau}} m_{\tau}\right),
\end{aligned}
$$

respectively, where $a=y_{1}^{*} v_{1}, b=y_{2}^{*} v_{1}, m_{e}=\left|y_{3} v_{1}\right|, m_{\mu}=\left|y_{4} v_{2}+y_{5} v_{3}\right|$, and $m_{\tau}=$ $\left|y_{4} v_{2}-y_{5} v_{3}\right|$, the $v_{j}$ being the vacuum expectation values (VEVs) of the lower components of the $\phi_{j}$.

Now consider the Majorana mass terms of the right-handed neutrinos,

$$
\mathcal{L}_{\text {Majorana }}=\frac{1}{2}\left(\nu_{e R}^{T}, \nu_{\mu R}^{T}, \nu_{\tau R}^{T}\right) C^{-1} M_{R}^{*}\left(\begin{array}{c}
\nu_{e R} \\
\nu_{\mu R} \\
\nu_{\tau R}
\end{array}\right)+\text { H.c. }
$$

They have dimension 3 and are, therefore, allowed to break the lepton-number symmetries $L_{\alpha}$. Because of the symmetry $\mathbb{Z}_{4}$, which is not allowed to be broken softly, one has

$$
M_{R}=M^{(\mathrm{AS})} .
$$

The seesaw mechanism [8, 9] prescribes

$$
\mathcal{M}_{\nu}=-M_{D}^{T} M_{R}^{-1} M_{D}
$$

hence

$$
\mathcal{M}_{\nu}^{-1}=-M_{D}^{-1} M_{R} M_{D}^{T^{-1}}
$$

It follows from equations (10) and (13) that $\mathcal{M}_{\nu}^{-1}$ is $\mu-\tau$ antisymmetric, just as $M_{R}$.

The model as it now stands is not realistic for many reasons, in particular because $M_{R}$ in equation (13) is not invertible and therefore the seesaw formula (14) cannot apply. One may correct for this by adding to the model one or more scalar $S U(2) \times U(1)$ invariants with non-vanishing lepton numbers. The simplest possibilities are the following:

1. A complex scalar $\chi_{e e}$ with $L_{e}=-2$ and $L_{\mu}=L_{\tau}=0$, which changes sign under $\mathbb{Z}_{4}$. Its Yukawa coupling $\chi_{e e} \nu_{e R}^{T} C^{-1} \nu_{e R}+$ H.c. generates a non-vanishing $\left(M_{R}\right)_{e e}$ upon $\chi_{e e}$ acquiring a VEV. One then has

$$
\text { case } 1: \quad \mathcal{M}_{\nu}^{-1}=\left(\begin{array}{ccc}
x & y & -y \\
y & z & 0 \\
-y & 0 & -z
\end{array}\right)
$$

2. A complex scalar $\chi_{\mu \tau}$ with $L_{e}=0$ and $L_{\mu}=L_{\tau}=-1$, which changes sign under $\mathbb{Z}_{4}$. Its Yukawa coupling $\chi_{\mu \tau} \nu_{\mu R}^{T} C^{-1} \nu_{\tau R}+$ H.c. generates a non-vanishing $\left(M_{R}\right)_{\mu \tau}$ upon $\chi_{\mu \tau}$ acquiring a VEV. One then has

$$
\text { case } 2: \quad \mathcal{M}_{\nu}^{-1}=\left(\begin{array}{ccc}
0 & y & -y \\
y & z & w \\
-y & w & -z
\end{array}\right) .
$$


3. Two complex scalars $\chi_{e \mu}$ and $\chi_{e \tau}, \chi_{e \mu}$ having $L_{e}=L_{\mu}=-1$ and $L_{\tau}=0$ while $\chi_{e \tau}$ has $L_{e}=L_{\tau}=-1$ and $L_{\mu}=0$. Under $\mathbb{Z}_{4}, \chi_{e \mu} \leftrightarrow-\chi_{e \tau}$. The Yukawa couplings $\nu_{e R}^{T} C^{-1}\left(\chi_{e \mu} \nu_{\mu R}+\chi_{e \tau} \nu_{\tau R}\right)+$ H.c. lead to $\left(M_{R}\right)_{e \mu} \neq-\left(M_{R}\right)_{e \tau}$ upon $\chi_{e \mu}$ and $\chi_{e \tau}$ acquiring $\mathrm{VEVs}$. One thus obtains

$$
\text { case } 3: \quad \mathcal{M}_{\nu}^{-1}=\left(\begin{array}{ccc}
0 & y & -y^{\prime} \\
y & z & 0 \\
-y^{\prime} & 0 & -z
\end{array}\right) \text {. }
$$

4. Two complex scalars $\chi_{\mu \mu}$ and $\chi_{\tau \tau}, \chi_{\mu \mu}$ having $L_{e}=L_{\tau}=0$ and $L_{\mu}=-2$ while $\chi_{\tau \tau}$ has $L_{e}=L_{\mu}=0$ and $L_{\tau}=-2$. Under $\mathbb{Z}_{4}, \chi_{\mu \mu} \leftrightarrow-\chi_{\tau \tau}$. The Yukawa couplings $\chi_{\mu \mu} \nu_{\mu R}^{T} C^{-1} \nu_{\mu R}+\chi_{\tau \tau} \nu_{\tau R}^{T} C^{-1} \nu_{\tau R}+$ H.c. lead to $\left(M_{R}\right)_{\mu \mu} \neq-\left(M_{R}\right)_{\tau \tau}$. One then has

$$
\text { case } 4: \quad \mathcal{M}_{\nu}^{-1}=\left(\begin{array}{ccc}
0 & y & -y \\
y & z & 0 \\
-y & 0 & -z^{\prime}
\end{array}\right) .
$$

One may put together any two of the possibilities 1-4 and obtain a neutrino mass matrix with more degrees of freedom-and correspondingly less predictive power. One thus obtains six more possibilities:

$$
\begin{aligned}
& \text { case } 5: \quad \mathcal{M}_{\nu}^{-1}=\left(\begin{array}{ccc}
x & y & -y \\
y & z & w \\
-y & w & -z
\end{array}\right) ; \\
& \text { case } 6: \quad \mathcal{M}_{\nu}^{-1}=\left(\begin{array}{ccc}
x & y & -y^{\prime} \\
y & z & 0 \\
-y^{\prime} & 0 & -z
\end{array}\right) \text {; } \\
& \text { case } 7: \quad \mathcal{M}_{\nu}^{-1}=\left(\begin{array}{ccc}
x & y & -y \\
y & z & 0 \\
-y & 0 & -z^{\prime}
\end{array}\right) \text {; } \\
& \text { case 8: } \quad \mathcal{M}_{\nu}^{-1}=\left(\begin{array}{ccc}
0 & y & -y^{\prime} \\
y & z & w \\
-y^{\prime} & w & -z
\end{array}\right) \text {; } \\
& \text { case } 9: \quad \mathcal{M}_{\nu}^{-1}=\left(\begin{array}{ccc}
0 & y & -y \\
y & z & w \\
-y & w & -z^{\prime}
\end{array}\right) \text {; } \\
& \text { case } 10: \quad \mathcal{M}_{\nu}^{-1}=\left(\begin{array}{ccc}
0 & y & -y^{\prime} \\
y & z & 0 \\
-y^{\prime} & 0 & -z^{\prime}
\end{array}\right) \text {. }
\end{aligned}
$$

It is the purpose of the rest of this paper to study, both analytically and numerically, whether cases 1-10 are viable or not, and to find out their predictions.

The neutrino mass matrix $\mathcal{M}_{\nu}$ may be rephased: $\mathcal{M}_{\nu} \rightarrow X \mathcal{M}_{\nu} X$, where $X$ is a diagonal unitary matrix. Because of this possibility, in general only three phases in the symmetric $\mathcal{M}_{\nu}$ are physically meaningful. Using this rephasing freedom, one finds that cases 1-4 have four physical parameters each, case 10 has five physical parameters, and the remaining cases 5-9 have six physical parameters each. 


\section{Cases 3, 4, and 10 are not viable}

These three cases contradict experiment since in all three of them the conditions

$$
\begin{aligned}
& \left(\mathcal{M}_{\nu}^{-1}\right)_{e e}=0 \\
& \left(\mathcal{M}_{\nu}^{-1}\right)_{\mu \tau}=0
\end{aligned}
$$

simultaneously hold [10]. Using

$$
\mathcal{M}_{\nu}^{-1}=U \operatorname{diag}\left(m_{1}^{-1}, m_{2}^{-1}, m_{3}^{-1}\right) U^{T},
$$

we find that the system of the two conditions (26) and (27) leads to

$$
\begin{aligned}
& \frac{m_{1}}{m_{3}}=\frac{U_{e 1} U_{\tau 2} U_{\tau 3}^{*}-U_{e 2} U_{\mu 1} U_{\mu 3}^{*}}{U_{e 2} U_{\tau 3} U_{\tau 1}^{*}-U_{e 3} U_{\mu 2} U_{\mu 1}^{*}}, \\
& \frac{m_{2}}{m_{3}}=\frac{U_{e 1} U_{\tau 2} U_{\tau 3}^{*}-U_{e 2} U_{\mu 1} U_{\mu 3}^{*}}{U_{e 3} U_{\tau 1} U_{\tau 2}^{*}-U_{e 1} U_{\mu 3} U_{\mu 2}^{*}} .
\end{aligned}
$$

We use the standard parametrization

$$
U=P_{F} \hat{U} P_{M},
$$

where

$$
\begin{gathered}
\hat{U}=\left(\begin{array}{ccc}
c_{13} c_{12} & c_{13} s_{12} & s_{13} e^{-i \delta} \\
-c_{23} s_{12}-s_{23} s_{13} c_{12} e^{i \delta} & c_{23} c_{12}-s_{23} s_{13} s_{12} e^{i \delta} & s_{23} c_{13} \\
-s_{23} s_{12}+c_{23} s_{13} c_{12} e^{i \delta} & s_{23} c_{12}+c_{23} s_{13} s_{12} e^{i \delta} & -c_{23} c_{13}
\end{array}\right), \\
P_{F}=\operatorname{diag}\left(e^{i \vartheta_{e}}, e^{i \vartheta_{\mu}}, e^{i \vartheta_{\tau}}\right), \\
P_{M}=\operatorname{diag}\left(e^{i \Theta / 2}, 1, e^{i \Omega / 2}\right) .
\end{gathered}
$$

In equation (32), $s_{k}=\sin \theta_{k}$ and $c_{k}=\cos \theta_{k}$ for $k=23,13,12$. The phases $\vartheta_{\alpha}$ in equation (33) are unobservable. Using the parametrization above, equations (29) and (30) translate into

$$
\begin{aligned}
& \frac{m_{1}}{m_{3}}=e^{i(\Theta-\Omega)} \frac{\left(1-|\epsilon|^{2}\right)\left[c_{23} s_{23}\left(s_{12}^{2}-c_{12}^{2}\right)+c_{12} s_{12}\left(s_{23}^{2}-c_{23}^{2}\right) \epsilon\right]}{c_{23} s_{23} s_{12}^{2}\left(1-2|\epsilon|^{2}\right)+c_{23} s_{23} c_{12}^{2} \epsilon^{* 2}+c_{12} s_{12}\left(c_{23}^{2}-s_{23}^{2}\right)|\epsilon|^{2} \epsilon^{*}}, \\
& \frac{m_{2}}{m_{3}}=e^{-i \Omega} \frac{\left(1-|\epsilon|^{2}\right)\left[c_{23} s_{23}\left(s_{12}^{2}-c_{12}^{2}\right)+c_{12} s_{12}\left(s_{23}^{2}-c_{23}^{2}\right) \epsilon\right]}{-c_{23} s_{23} c_{12}^{2}\left(1-2|\epsilon|^{2}\right)-c_{23} s_{23} s_{12}^{2} \epsilon^{* 2}+c_{12} s_{12}\left(c_{23}^{2}-s_{23}^{2}\right)|\epsilon|^{2} \epsilon^{*}}
\end{aligned}
$$

where we have defined $\epsilon \equiv s_{13} e^{i \delta}$. Experimentally [1], both $|\epsilon|$ and $\left|c_{23}^{2}-s_{23}^{2}\right|$ are at most

of order 0.2; neglecting terms of order $\epsilon^{2},\left(c_{23}^{2}-s_{23}^{2}\right)^{2}$, and $\epsilon\left(c_{23}^{2}-s_{23}^{2}\right)$ in equations (35) and (36), one obtains

$$
\begin{aligned}
& \frac{m_{1}}{m_{3}} \approx 1-\frac{c_{12}^{2}}{s_{12}^{2}}, \\
& \frac{m_{2}}{m_{3}} \approx 1-\frac{s_{12}^{2}}{c_{12}^{2}} .
\end{aligned}
$$


Using the experimental result [1] $\tan ^{2} \theta_{12} \approx 0.43$, one obtains

$$
\frac{m_{2}^{2}-m_{1}^{2}}{m_{3}^{2}-m_{1}^{2}} \approx 1.9 .
$$

Although the number in the right-hand side of equation (39) is rather sensitive to the precise value of $\tan ^{2} \theta_{12}$, its order of magnitude does not change. It is in manifest contradiction with experiment, which gives

$$
\left|\frac{m_{2}^{2}-m_{1}^{2}}{m_{3}^{2}-m_{1}^{2}}\right| \approx 0.037 .
$$

Thus, cases 3, 4, and 10 are excluded.

\section{Case 1 is not viable}

Define the orthogonal matrix

$$
R_{1}=\left(\begin{array}{ccc}
1 & 0 & 0 \\
0 & r & r \\
0 & -r & r
\end{array}\right), \text { where } r=\frac{1}{\sqrt{2}} .
$$

Then, with the $\mathcal{M}_{\nu}^{-1}$ of case 1 , in equation (16), one has

$$
R_{1}^{T} \mathcal{M}_{\nu}^{-1} R_{1}=\left(\begin{array}{ccc}
x & \sqrt{2} y & 0 \\
\sqrt{2} y & 0 & z \\
0 & z & 0
\end{array}\right) .
$$

This is a mass matrix of the Fritzsch type, which may be diagonalized in a standard fashion [11. The three phases in the matrix (42) may be discarded by using a diagonal unitary matrix $P_{1}$ :

$$
P_{1} R_{1}^{T} \mathcal{M}_{\nu}^{-1} R_{1} P_{1}=\left(\begin{array}{ccc}
|x| & \sqrt{2}|y| & 0 \\
\sqrt{2}|y| & 0 & |z| \\
0 & |z| & 0
\end{array}\right) .
$$

This matrix has eigenvalues $\lambda_{1},-\lambda_{2}$, and $\lambda_{3}$, with $\lambda_{1}>\lambda_{2}>\lambda_{3}>0$. If we define the diagonal unitary matrix

$$
P_{2}=\operatorname{diag}(1, i, 1),
$$

then there is an orthogonal matrix $R_{2}$ such that

$$
P_{2} R_{2}^{T} P_{1} R_{1}^{T} \mathcal{M}_{\nu}^{-1} R_{1} P_{1} R_{2} P_{2}=\operatorname{diag}\left(\lambda_{3}, \lambda_{2}, \lambda_{1}\right) .
$$

The standard diagonalization of the Fritzsch mass matrix yields

$$
\begin{aligned}
& \left(R_{2}\right)_{11}=\sqrt{\frac{\lambda_{3}\left(\lambda_{1}+\lambda_{3}\right)\left(\lambda_{2}-\lambda_{3}\right)}{\left(\lambda_{1}-\lambda_{3}\right)\left(\lambda_{2}+\lambda_{3}\right)\left(\lambda_{1}-\lambda_{2}+\lambda_{3}\right)}}, \\
& \left(R_{2}\right)_{12}=\sqrt{\frac{\lambda_{2}\left(\lambda_{1}-\lambda_{2}\right)\left(\lambda_{2}-\lambda_{3}\right)}{\left(\lambda_{1}+\lambda_{2}\right)\left(\lambda_{2}+\lambda_{3}\right)\left(\lambda_{1}-\lambda_{2}+\lambda_{3}\right)}}, \\
& \left(R_{2}\right)_{13}=\sqrt{\frac{\lambda_{1}\left(\lambda_{1}-\lambda_{2}\right)\left(\lambda_{1}+\lambda_{3}\right)}{\left(\lambda_{1}+\lambda_{2}\right)\left(\lambda_{1}-\lambda_{3}\right)\left(\lambda_{1}-\lambda_{2}+\lambda_{3}\right)}} .
\end{aligned}
$$


Since

$$
U^{\dagger} \mathcal{M}_{\nu}^{-1} U^{*}=\operatorname{diag}\left(m_{1}^{-1}, m_{2}^{-1}, m_{3}^{-1}\right),
$$

we see that there are two possibilities:

1. If the neutrino mass spectrum is normal, $m_{1}<m_{2}<m_{3}$, then $\lambda_{j}=m_{j}^{-1}$ for $j=1,2,3$ and

$$
\left|\frac{U_{e 2}}{U_{e 1}}\right|^{2}=\left[\frac{\left(R_{2}\right)_{12}}{\left(R_{2}\right)_{13}}\right]^{2}=\frac{m_{2}^{-1}\left(m_{2}^{-1}-m_{3}^{-1}\right)\left(m_{1}^{-1}-m_{3}^{-1}\right)}{m_{1}^{-1}\left(m_{1}^{-1}+m_{3}^{-1}\right)\left(m_{2}^{-1}+m_{3}^{-1}\right)} .
$$

2. If the neutrino mass spectrum is inverted, $m_{3}<m_{1}<m_{2}$, then $\lambda_{1}=m_{3}^{-1}, \lambda_{2}=$ $m_{1}^{-1}$, and $\lambda_{3}=m_{2}^{-1}$. One then has

$$
\left|\frac{U_{e 2}}{U_{e 1}}\right|^{2}=\left[\frac{\left(R_{2}\right)_{11}}{\left(R_{2}\right)_{12}}\right]^{2}=\frac{m_{2}^{-1}\left(m_{3}^{-1}+m_{2}^{-1}\right)\left(m_{3}^{-1}+m_{1}^{-1}\right)}{m_{1}^{-1}\left(m_{3}^{-1}-m_{1}^{-1}\right)\left(m_{3}^{-1}-m_{2}^{-1}\right)} .
$$

The inverted case is readily excluded. With $m_{2}^{2}-m_{1}^{2}=8.1 \times 10^{-5} \mathrm{eV}^{2}$ and $\left|m_{1}^{2}-m_{3}^{2}\right|=$ $2.2 \times 10^{-3} \mathrm{eV}^{2}$, one finds that equation (151) yields $\left|U_{e 2} / U_{e 1}\right|^{2}>0.98$ for all $m_{1}$, which is far above the best-fit value $\left|U_{e 2} / U_{e 1}\right|^{2}=0.43$. As for equation (50), with the above values for the mass-squared differences, it always yields $\left|U_{e 2} / U_{e 1}\right|^{2}<0.28$. This value translates into $s_{12}^{2}<0.22$. Reporting here for further use the $3 \sigma$ values derived in [1],

$$
\begin{aligned}
& 7.2 \times 10^{-5} \mathrm{eV}^{2}<m_{2}^{2}-m_{1}^{2}<9.1 \times 10^{-5} \mathrm{eV}^{2}, \\
& 1.4 \times 10^{-3} \mathrm{eV}^{2}<\left|m_{1}^{2}-m_{3}^{2}\right|<3.3 \times 10^{-3} \mathrm{eV}^{2}, \\
& 0.23<\sin ^{2} \theta_{12}<0.38, \\
& 0.34<\sin ^{2} \theta_{23}<0.66, \\
& \sin ^{2} \theta_{13}<0.047,
\end{aligned}
$$

we see that 0.22 is just outside the $3 \sigma$ range of $\sin ^{2} \theta_{12}$. However, the upper bound on $\left|U_{e 2} / U_{e 1}\right|^{2}$ of equation (50) is sensitive to the values of the mass-squared differences. Taking the lower limit $m_{2}^{2}-m_{1}^{2}=7.2 \times 10^{-5} \mathrm{eV}^{2}$ from inequality (52) and the upper limit $m_{3}^{2}-m_{1}^{2}=3.3 \times 10^{-3} \mathrm{eV}^{2}$ from inequality (53), the upper bound on $\left|U_{e 2} / U_{e 1}\right|^{2}$ is 0.35 , or $s_{12}^{2}<0.26$, which is inside the $3 \sigma$ range of inequality (54). In that case, a simultaneous consideration of $s_{13}^{2}=\left[\left(R_{2}\right)_{11}\right]^{2}$ leads to $s_{13}^{2}=0.29$, for the $m_{1}$ where the maximum of equation (50) is reached. In view of the inequality (56), this value is clearly much too large, hence case 1 is excluded.

\section{Case 2 is not viable}

Case 2 is defined by

$$
\begin{aligned}
\left(\mathcal{M}_{\nu}^{-1}\right)_{e e} & =0 \\
\left(\mathcal{M}_{\nu}^{-1}\right)_{e \mu}+\left(\mathcal{M}_{\nu}^{-1}\right)_{e \tau} & =0 \\
\left(\mathcal{M}_{\nu}^{-1}\right)_{\mu \mu}+\left(\mathcal{M}_{\nu}^{-1}\right)_{\tau \tau} & =0
\end{aligned}
$$


In the following, we shall use the quantities

$$
\mu_{1} \equiv m_{1} e^{-i \Theta}, \quad \mu_{2} \equiv m_{2}, \quad \mu_{3} \equiv m_{3} e^{-i \Omega} .
$$

The matrix elements $\left(\mathcal{M}_{\nu}^{-1}\right)_{\alpha \beta}$ are then given as

$$
\left(\mathcal{M}_{\nu}^{-1}\right)_{\alpha \beta}=e^{i\left(\vartheta_{\alpha}+\vartheta_{\beta}\right)} \sum_{j=1}^{3} \frac{\hat{U}_{\alpha j} \hat{U}_{\beta j}}{\mu_{j}},
$$

with $\hat{U}$ in equation (32). We shall moreover use

$$
\beta \equiv \vartheta_{\tau}-\vartheta_{\mu}
$$

$\epsilon \equiv s_{13} \exp (i \delta)$, and $\nu \equiv \cos 2 \theta_{23}$. We also introduce the small parameter $\lambda=0.22$ which is useful since, experimentally, both $|\epsilon|$ and $|\nu|$ have upper bounds of order $\lambda$. Moreover, $\left(m_{2}^{2}-m_{1}^{2}\right) /\left|m_{1}^{2}-m_{3}^{2}\right| \approx 0.037$ is of order $\lambda^{2}$.

Equation (57) yields

$$
\frac{\left(\hat{U}_{e 1}\right)^{2}}{\mu_{1}}+\frac{\left(\hat{U}_{e 2}\right)^{2}}{\mu_{2}}+\frac{\left(\hat{U}_{e 3}\right)^{2}}{\mu_{3}}=0
$$

or

$$
\mu_{3}=-\frac{\epsilon^{* 2}}{1-|\epsilon|^{2}}\left(\frac{c_{12}^{2}}{\mu_{1}}+\frac{s_{12}^{2}}{\mu_{2}}\right)^{-1} .
$$

Taking into account that $m_{2}>m_{1}$ and $c_{12}>s_{12}$, equation (64) leads to the inequality

$$
\frac{m_{3}}{m_{1}}<\frac{|\epsilon|^{2}}{1-|\epsilon|^{2}} \frac{1}{c_{12}^{2}-s_{12}^{2}},
$$

hence the neutrino mass spectrum in case 2 must be inverted. The quantity $c_{12}^{2}-s_{12}^{2}$ is smaller than 1: with the best-fit value $s_{12}^{2}=0.30$, it is 0.40 ; at the upper edge of the $3 \sigma$ range for $s_{12}^{2}$, it is only 0.24 , i.e. of order $\lambda$. Thus, it is appropriate to admit $m_{3} / m_{1}$ to be of order $\lambda$, instead of $\lambda^{2}$. As for $m_{3} / m_{2}$, it is of the same order as $m_{3} / m_{1}$, since

$$
\frac{m_{3}}{m_{2}}=\frac{m_{3}}{\sqrt{m_{1}^{2}+\Delta m_{\odot}^{2}}}
$$

and $m_{1}^{2}$ is of the order of the atmospheric mass-squared difference when $m_{3} / m_{1}$ is very small. We obtain a picture in which $m_{1} \approx m_{2} \approx \sqrt{\Delta m_{\text {atm }}^{2}}$, while $m_{3} / m_{1} \lesssim 0.2$ is small.

Equation (58) gives, after using equation (64),

$$
s_{23}-e^{i \beta} c_{23}+\zeta\left(c_{23}+e^{i \beta} s_{23}\right)=0
$$

where

$$
\zeta \equiv \epsilon^{*} \frac{\mu_{2}-\mu_{1}}{c_{12}^{2} \mu_{2}+s_{12}^{2} \mu_{1}} c_{12} s_{12}
$$

Next we address equation (59). Defining

$$
\begin{aligned}
z & \equiv c_{23}^{2} e^{i \beta}+s_{23}^{2} e^{-i \beta} \\
& =\cos \beta+i \nu \sin \beta
\end{aligned}
$$


we find that equation (59) may be written as

$$
a z+b z^{*}+c=0
$$

where

$$
\begin{aligned}
a & =1-2|\epsilon|^{2}, \\
b & =-\epsilon^{* 2} \frac{s_{12}^{2} \mu_{2}+c_{12}^{2} \mu_{1}}{c_{12}^{2} \mu_{2}+s_{12}^{2} \mu_{1}}, \\
c & =2 i|\epsilon|^{2} \sqrt{1-\nu^{2}} \zeta \sin \beta,
\end{aligned}
$$

where we have once again used equation (64) and the definition (68). The solution of equation (170) for $z$ is

$$
z=\frac{b c^{*}-a^{*} c}{|a|^{2}-|b|^{2}} .
$$

An upper limit on the absolute value of $\zeta$ is

$$
\begin{aligned}
|\zeta| & \leq|\epsilon| \frac{m_{2}+m_{1}}{c_{12}^{2} m_{2}-s_{12}^{2} m_{1}} c_{12} s_{12} \\
& =|\epsilon| \frac{2\left(m_{2}+m_{1}\right)}{\left(c_{12}^{2}-s_{12}^{2}\right)\left(m_{1}+m_{2}\right)+m_{2}-m_{1}} c_{12} s_{12} \\
& <|\epsilon| \tan 2 \theta_{12} .
\end{aligned}
$$

The $3 \sigma$ limits $|\epsilon|<0.22$ and $\tan 2 \theta_{12}<4.04$, cf. inequalities (54) and (156), lead to $|\zeta|<0.89$; at the $2 \sigma$ level - see [1] this bound is already down to $|\zeta|<0.50$.

Let us now use simple approximations to show that case 2 is not viable. Since $a \approx 1$ and $|b| \lesssim \lambda^{2}$, we conclude from equation (74) that $z \simeq-c$ :

$$
\cos \beta+i \nu \sin \beta \simeq-2 i|\epsilon|^{2} \sqrt{1-\nu^{2}} \zeta \sin \beta .
$$

The right-hand side of equation (76) being at most of order $\lambda^{2}$, one must have

$$
e^{i \beta} \simeq \eta i(\eta= \pm 1), \quad \nu \simeq 0
$$

up to corrections of order $\lambda^{2}$. Therefore,

$$
c_{23}+e^{i \beta} s_{23} \simeq \frac{1+\eta i}{\sqrt{2}}, \quad s_{23}-e^{i \beta} c_{23} \simeq \frac{1-\eta i}{\sqrt{2}} .
$$

Introducing the approximations (78) into equation (67), one finds

$$
\zeta \simeq \frac{-1+i \eta}{1+i \eta}=i \eta
$$

i.e. $\zeta= \pm i$. But we know that at $3 \sigma$ level $|\zeta|$ is at most 0.89 . Hence case 2 is ruled out. 


\section{Cases 5-9 are viable}

Case 5 is defined by conditions (58) and (59). Using the phase $\beta$ of the definition (62), they read

$$
\begin{array}{r}
\frac{\hat{U}_{e 1}\left(\hat{U}_{\mu 1}+e^{i \beta} \hat{U}_{\tau 1}\right)}{\mu_{1}}+\frac{\hat{U}_{e 2}\left(\hat{U}_{\mu 2}+e^{i \beta} \hat{U}_{\tau 2}\right)}{\mu_{2}}+\frac{\hat{U}_{e 3}\left(\hat{U}_{\mu 3}+e^{i \beta} \hat{U}_{\tau 3}\right)}{\mu_{3}}=0, \\
\frac{\left[\left(\hat{U}_{\mu 1}\right)^{2}+e^{2 i \beta}\left(\hat{U}_{\tau 1}\right)^{2}\right]}{\mu_{1}}+\frac{\left[\left(\hat{U}_{\mu 2}\right)^{2}+e^{2 i \beta}\left(\hat{U}_{\tau 2}\right)^{2}\right]}{\mu_{2}}+\frac{\left[\left(\hat{U}_{\mu 3}\right)^{2}+e^{2 i \beta}\left(\hat{U}_{\tau 3}\right)^{2}\right]}{\mu_{3}}=0 .
\end{array}
$$

These two equations lead to

$$
\begin{aligned}
\frac{\mu_{1}}{\mu_{3}}=\frac{\hat{U}_{e 2}\left(\hat{U}_{\mu 2}+e^{i \beta} \hat{U}_{\tau 2}\right)\left(\hat{U}_{\mu 1}^{2}+e^{2 i \beta} \hat{U}_{\tau 1}^{2}\right)-\hat{U}_{e 1}\left(\hat{U}_{\mu 1}+e^{i \beta} \hat{U}_{\tau 1}\right)\left(\hat{U}_{\mu 2}^{2}+e^{2 i \beta} \hat{U}_{\tau 2}^{2}\right)}{\hat{U}_{e 3}\left(\hat{U}_{\mu 3}+e^{i \beta} \hat{U}_{\tau 3}\right)\left(\hat{U}_{\mu 2}^{2}+e^{2 i \beta} \hat{U}_{\tau 2}^{2}\right)-\hat{U}_{e 2}\left(\hat{U}_{\mu 2}+e^{i \beta} \hat{U}_{\tau 2}\right)\left(\hat{U}_{\mu 3}^{2}+e^{2 i \beta} \hat{U}_{\tau 3}^{2}\right)}, \\
\frac{\mu_{2}}{\mu_{3}}=\frac{\hat{U}_{e 2}\left(\hat{U}_{\mu 2}+e^{i \beta} \hat{U}_{\tau 2}\right)\left(\hat{U}_{\mu 1}^{2}+e^{2 i \beta} \hat{U}_{\tau 1}^{2}\right)-\hat{U}_{e 1}\left(\hat{U}_{\mu 1}+e^{i \beta} \hat{U}_{\tau 1}\right)\left(\hat{U}_{\mu 2}^{2}+e^{2 i \beta} \hat{U}_{\tau 2}^{2}\right)}{\hat{U}_{e 1}\left(\hat{U}_{\mu 1}+e^{i \beta} \hat{U}_{\tau 1}\right)\left(\hat{U}_{\mu 3}^{2}+e^{2 i \beta} \hat{U}_{\tau 3}^{2}\right)-\hat{U}_{e 3}\left(\hat{U}_{\mu 3}+e^{i \beta} \hat{U}_{\tau 3}\right)\left(\hat{U}_{\mu 1}^{2}+e^{2 i \beta} \hat{U}_{\tau 1}^{2}\right)} .
\end{aligned}
$$

In each of cases 6-9 there is a set of two conditions of the type $\left(\mathcal{M}_{\nu}^{-1}\right)_{\alpha \beta}=0$ and $\left(\mathcal{M}_{\nu}^{-1}\right)_{\gamma \delta}+\left(\mathcal{M}_{\nu}^{-1}\right)_{\rho \sigma}=0$. By solving the system of these two equations one obtains

$$
\begin{aligned}
& \frac{\mu_{1}}{\mu_{3}}= \frac{\left(\hat{U}_{\gamma 2} \hat{U}_{\delta 2}+e^{i \varphi} \hat{U}_{\rho 2} \hat{U}_{\sigma 2}\right) \hat{U}_{\alpha 1} \hat{U}_{\beta 1}-\left(\hat{U}_{\gamma 1} \hat{U}_{\delta 1}+e^{i \varphi} \hat{U}_{\rho 1} \hat{U}_{\sigma 1}\right) \hat{U}_{\alpha 2} \hat{U}_{\beta 2}}{\left(\hat{U}_{\gamma 3} \hat{U}_{\delta 3}+e^{i \varphi} \hat{U}_{\rho 3} \hat{U}_{\sigma 3}\right) \hat{U}_{\alpha 2} \hat{U}_{\beta 2}-\left(\hat{U}_{\gamma 2} \hat{U}_{\delta 2}+e^{i \varphi} \hat{U}_{\rho 2} \hat{U}_{\sigma 2}\right) \hat{U}_{\alpha 3} \hat{U}_{\beta 3}} \\
& \frac{\mu_{2}}{\mu_{3}}=\frac{\left(\hat{U}_{\gamma 2} \hat{U}_{\delta 2}+e^{i \varphi} \hat{U}_{\rho 2} \hat{U}_{\sigma 2}\right) \hat{U}_{\alpha 1} \hat{U}_{\beta 1}-\left(\hat{U}_{\gamma 1} \hat{U}_{\delta 1}+e^{i \varphi} \hat{U}_{\rho 1} \hat{U}_{\sigma 1}\right) \hat{U}_{\alpha 2} \hat{U}_{\beta 2}}{\left(\hat{U}_{\gamma 1} \hat{U}_{\delta 1}+e^{i \varphi} \hat{U}_{\rho 1} \hat{U}_{\sigma 1}\right) \hat{U}_{\alpha 3} \hat{U}_{\beta 3}-\left(\hat{U}_{\gamma 3} \hat{U}_{\delta 3}+e^{i \varphi} \hat{U}_{\rho 3} \hat{U}_{\sigma 3}\right) \hat{U}_{\alpha 1} \hat{U}_{\beta 1}}
\end{aligned}
$$

where $\varphi \equiv \vartheta_{\rho}+\vartheta_{\sigma}-\vartheta_{\gamma}-\vartheta_{\delta}$. The unphysical phase $\varphi$ arises because the relative phase in the equality $\left(\mathcal{M}_{\nu}^{-1}\right)_{\gamma \delta}=-\left(\mathcal{M}_{\nu}^{-1}\right)_{\rho \sigma}$ is arbitrary due to the rephasing freedom.

The right-hand sides of equations (82)-(85) are functions of the observables $\theta_{12}, \theta_{23}$, $\theta_{13}$, and $\delta$, plus an unphysical phase $\beta$ in equations (82) and (83), $\varphi$ in equations (84) and (85).

We have used a numerical approach to cases 5-9, similar to the one in [12]. We have numerically generated random parameter sets of the four observables $\theta_{12}, \theta_{23}, \theta_{13}$, and $\delta$, of the unphysical phase, and of the mass $m_{3}$. For each of these sets, the neutrino mass ratios $m_{1} / m_{3}$ and $m_{2} / m_{3}$ have been computed by taking the absolute values in the corresponding equations for $\mu_{1} / \mu_{3}$ and $\mu_{2} / \mu_{3}$; by taking the absolute values of those equations we avoid considering the two observables on which we have hardly any experimental grip - the Majorana phases $\Theta$ and $\Omega$. Whenever the obtained neutrino mass ratios, together with the inputted $m_{3}$, were consistent with the experimental values for $m_{2}^{2}-m_{1}^{2}$ and $\left|m_{1}^{2}-m_{3}^{2}\right|$, we plotted the corresponding physical point in a scatter plot. The resulting scatter plots of the allowed regions for each model are presented in figures 15. We have generated 100,000 random parameter sets for each of the cases 5, 6, 7, and 9; for case 8 we have used $2,000,000$ random parameter sets because we have found that only a very small percentage of them turned out to be allowed. 
We present in figures 115, for each of cases 5-9, respectively, two types of scatter plots of the allowed regions: in the $\sin ^{2} 2 \theta_{23}-\left|U_{e 3}\right|$ plane, and in the $m_{2}-m_{3}$ plane.

The neutrino mass spectrum in cases 5 and 7 may be of any possible type: normal, inverted, or quasi-degenerate. Case 6 typically displays a normal spectrum $\left(m_{3}>m_{2}\right)$. Cases 8 and 9 have inverted neutrino mass spectra $\left(m_{3}<m_{2}\right)$, as derived already in section $\left[5\right.$ from the condition $\left(\mathcal{M}_{\nu}^{-1}\right)_{e e}=0$ alone. Notice that case 8 has a distinct prediction: it requires the atmospheric neutrino mixing to be very close to maximal, and $\left|U_{e 3}\right|$ is also usually close to its upper bound; this, too, we had already anticipated analytically in section 5

For all cases $5-9,\left|U_{e 3}\right|$ cannot vanish, but it may be very small. We have performed a dedicated search of points with very small $\left|U_{e 3}\right|$ by constructing random parameter sets with very small inputted $\theta_{13}$. We thereby found the lower bounds on $\left|U_{e 3}\right|$ given in table 1. One sees that, in all cases but in case $8,\left|U_{e 3}\right|$ can attain values so low as to be

\begin{tabular}{|c|c|c|c|c|c|}
\hline & case 5 & case 6 & case 7 & case 8 & case 9 \\
\hline$\left|U_{e 3}\right|>$ & 0.001 & $10^{-6}$ & 0.0005 & 0.04 & 0.0003 \\
\hline$\langle m\rangle_{e e}(\mathrm{eV})>$ & 0.0001 & $10^{-5}$ & 0.0001 & 0.01 & 0.01 \\
\hline
\end{tabular}

Table 1: Lower bounds of $\left|U_{e 3}\right|$ and on $\langle m\rangle_{e e}$, for each case.

experimentally undistinguishable from zero.

We have also investigated the decay rate for neutrinoless double-beta decay in each model. That rate is controlled by the effective Majorana mass

$$
\langle m\rangle_{e e}=\left|m_{1} c_{13}^{2} c_{12}^{2} e^{i \Theta}+m_{2} c_{13}^{2} s_{12}^{2}+m_{3} s_{13}^{2} e^{i(\Omega-2 \delta)}\right| .
$$

The values of the Majorana phases are extracted from the equations for $\mu_{1} / \mu_{3}$ and $\mu_{2} / \mu_{3}$. We present scatter plots of $\langle m\rangle_{e e}$ as a function of $m_{2}$, for each case, in figures [6] and 7 . One sees in those figures that $\langle m\rangle_{e e}$ is at most $0.3 \mathrm{eV}$ in cases $5-7$; in cases 8 and 9 the upper bound is about an order of magnitude smaller. Lower bounds of $\langle m\rangle_{e e}$ are given in table 1, since cases 8 and 9 have an inverted neutrino mass spectrum, the lower bound on $\langle m\rangle_{e e}$ is not so suppressed there as in cases $5-7$.

\section{Summary}

The type-I seesaw mechanism has three sources of lepton mixing: the charged-lepton mass matrix $M_{\ell}$, the neutrino Dirac mass matrix $M_{D}$, and the mass matrix $M_{R}$ of the right-handed neutrinos. It is interesting to choose $M_{R}$ as the only source of mixing. A symmetry reason for this choice is provided by the assumption of conservation of the family lepton numbers $L_{\alpha}(\alpha=e, \mu, \tau)$ in all terms of dimension 4 in the Lagrangian, because in that case $M_{\ell}$ and $M_{D}$ are automatically diagonal; the Majorana mass terms of the right-handed neutrinos break the $L_{\alpha}$ softly and we are therefore allowed to obtain lepton mixing from $M_{R}$ [7]. This framework admits an arbitrary number of Higgs doublets, as their Yukawa-coupling matrices are all diagonal, hence flavour-changing Yukawa neutral 
interactions among the charged leptons are strongly suppressed [13. In this framework, it looks sensible to consider relations among the matrix elements of the inverted neutrino mass matrix $\mathcal{M}_{\nu}^{-1}[10$.

In this paper, we have used as a starting point a $\mu-\tau$ interchange antisymmetry in $\mathcal{M}_{\nu}^{-1}$. We have shown that this can be obtained by imposing on the full Lagrangian a symmetry of the $\mathbb{Z}_{4}$ type. However, such a $\mu-\tau$ antisymmetric matrix $\mathcal{M}_{\nu}^{-1}$ is singular, therefore $\mu-\tau$ symmetric "perturbations" must be added to it. We have done so by introducing complex scalar $S U(2) \times U(1)$ invariants with Yukawa couplings to the righthanded neutrinos. These scalar fields have non-trivial lepton numbers $L_{\alpha}$, and there are four basic possibilities to introduce such singlets in accord with the above-mentioned $\mathbb{Z}_{4}$ - see section 2 . The VEVs of these scalar $S U(2) \times U(1)$ invariants generate the $\mu-\tau$ symmetric "perturbations" in $\mathcal{M}_{\nu}^{-1}$.

Each of the four basic possibilities leads to a four-parameter neutrino mass matrix (cases 1, 2, 3, and 4 in section 2) which we have shown to be ruled out by the data. As a next step, we have considered the six combinations of the four basic possibilities. One of them leads to a five-parameter mass matrix (case 10) and is ruled out as well. On the other hand, the remaining cases 5, 6, 7, 8, and 9, which have neutrino mass matrices with six parameters, are physically viable, as we have shown by numerical analysis. We have refrained from combining more than two of the four basic possibilities, since the ensuing neutrino mass matrices presumably have negligible predictive power.

The five viable cases have in common that they allow $s_{13}^{2}$ to be as large as the present upper bound. The numerical scan in the parameter space of cases 5, 6, and 7 shows a preference for a hierarchical spectrum, although quasi-degenerate or inverted spectra cannot be ruled out. The neutrino mass spectrum in cases 8 and 9 has inverted hierarchy; this follows solely from the condition $\left(\mathcal{M}_{\nu}^{-1}\right)_{e e}=0$, which has been analytically analyzed in section 5 , an analysis which is borne out by our numerical results - see figures 4 and 5 . The most predictive neutrino mass matrix is the one of case 8 , because it leads to practically maximal atmospheric mixing, correlated with a large $s_{13}^{2}$ - see the analytical discussion of conditions (57) and (59) in section 5, and the numerical result in figure 4 .

The research presented in this paper displays once again the usefulness of conditions on the matrix elements of the inverted neutrino mass matrix $\mathcal{M}_{\nu}^{-1}$. Indeed, in the framework employed here, it is most natural that symmetries imposed on the Lagrangian lead to relations in $\mathcal{M}_{\nu}^{-1}$. In this paper we have used $\mu-\tau$ interchange antisymmetry, instead of the well-known $\mu-\tau$ interchange symmetry, as the basic ingredient to obtain viable and predictive neutrino mass matrices.

Acknowledgements The work of S.K. was supported by the Japanese Society for the Promotion of Science. The work of L.L. was supported by the Portuguese Fundação para a Ciência e a Tecnologia through the projects POCTI/FNU/44409/2002 and U777Plurianual. The work of M.T. is supported by the Grant-in-Aid for Science Research of the Ministry of Education, Science, and Culture of Japan No. 16028205, No. 17540243. 


\section{References}

[1] M. Maltoni, T. Schwetz, M. Tortola and J.W.F. Valle, Status of global fits to neutrino oscillations, New J. Phys. 6 (2004) 122 hep-ph/0405172.

[2] G.L. Fogli, E. Lisi, A. Marrone and A. Palazzo, Global analysis of three-flavor neutrino masses and mixings, hep-ph/0506083.

[3] G. Altarelli and F. Feruglio, Models of neutrino masses and mixings, New J. Phys. 6 (2004) 106 hep-ph/0405048;

G. Altarelli, Normal and special models of neutrino masses and mixings, to be published in the Proceedings of 19th Rencontres de physique de la vallée d'Aoste: Results and perspectives in particle physics, La Thuile, Aosta valley, Italy, 27 Feb.5 Mar. 2005 hep-ph/0508053;

R.N. Mohapatra et al., Theory of neutrinos: A white paper, hep-ph/0510213.

[4] T. Fukuyama and H. Nishiura, Mass matrix of Majorana neutrinos, hep-ph/9702253.

E. Ma and M. Raidal, Neutrino mass, muon anomalous magnetic moment, and lepton flavor nonconservation, Phys. Rev. Lett. 87 (2001) 011802 hep-ph/0102255; Err. ibid. 87 (2001) 159901;

C.S. Lam, A 2-3 symmetry in neutrino oscillations, Phys. Lett. B 507 (2001) 214 hep-ph/0104116;

K.R.S. Balaji, W. Grimus and T. Schwetz, The solar LMA neutrino oscillation solution in the Zee model, Phys. Lett. B 508 (2001) 301 hep-ph/0104035;

E. Ma, The all-purpose neutrino mass matrix, Phys. Rev. D 66 (2002) 117301 hep-ph/0207352;

P.F. Harrison and W.G. Scott, $\mu-\tau$ reflection symmetry in lepton mixing and neutrino oscillations, Phys. Lett. B 547 (2002) 219 hep-ph/0210197.

[5] For specific schemes and additional references, see for instance

Y. Koide, Universal texture of quark and lepton mass matrices with an extended flavor $2 \leftrightarrow 3$ symmetry, Phys. Rev. D 69 (2004) 093001 hep-ph/0312207;

W. Grimus, A.S. Joshipura, S. Kaneko, L. Lavoura, H. Sawanaka and M. Tanimoto, Non-vanishing $U_{e 3}$ and $\cos 2 \theta_{23}$ from a broken $Z_{2}$ symmetry, Nucl. Phys. B 713 (2005) 151 hep-ph/0408123;

W. Grimus and L. Lavoura, $S_{3} \times \mathbb{Z}_{2}$ model for neutrino mass matrices, JHEP 08 (2005) 013 hep-ph/0504153;

R.N. Mohapatra and W. Rodejohann, Broken $\mu-\tau$ symmetry and leptonic $C P$ violation, Phys. Rev. D 72 (2005) 053001 hep-ph/0507312.

[6] T. Kitabayashi and M. Yasue, $\mu-\tau$ symmetry and maximal CP violation, Phys. Lett. B 621 (2005) 133 hep-ph/0504212;

I. Aizawa, T. Kitabayashi and M. Yasue, Determination of neutrino mass texture for maximal CP violation, Nucl. Phys. B 728 (2005) 220 hep-ph/0507332;

T. Kitabayashi and M. Yasue, A new type of complex neutrino mass texture and $\mu-\tau$ symmetry, hep-ph/0510132 
[7] W. Grimus and L. Lavoura, Softly broken lepton numbers and maximal neutrino mixing, JHEP 07 (2001) 045 hep-ph/0105212.

[8] P. Minkowski, $\mu \rightarrow e \gamma$ at a rate of one out of $10^{9}$ muon decays?, Phys. Lett. B 67 (1977) 421;

T. Yanagida, in Proceedings of the workshop on unified theory and baryon number in the universe, O. Sawata and A. Sugamoto eds., KEK report 79-18, Tsukuba, Japan 1979 ;

S.L. Glashow, in Quarks and leptons, proceedings of the advanced study institute (Cargèse, Corsica, 1979), J.-L. Basdevant et al. eds., Plenum, New York 1981;

M. Gell-Mann, P. Ramond and R. Slansky, Complex spinors and unified theories, in Supergravity, D.Z. Freedman and F. van Nieuwenhuizen eds., North Holland, Amsterdam 1979;

R.N. Mohapatra and G. Senjanovic, Neutrino mass and spontaneous parity violation, Phys. Rev. Lett. 44 (1980) 912.

[9] For detailed discussions of the seesaw mechanism, see

J. Schechter and J.W.F. Valle, Neutrino masses in $S U(2) \times U(1)$ theories, Phys. Rev. D 22 (1980) 2227;

S.M. Bilenky, J. Hosek and S.T. Petcov, On oscillations of neutrinos with Dirac and Majorana masses, Phys. Lett. 94B (1980) 495;

I.Yu. Kobzarev, B.V. Martemyanov, L.B. Okun and M.G. Shchepkin, The phenomenology of neutrino oscillations, Yad. Phys. 32 (1980) 1590 [Sov. J. Nucl. Phys. 32 (1981) 823];

J. Schechter and J.W.F. Valle, Neutrino decay and spontaneous violation of lepton number, Phys. Rev. D 25 (1982) 774;

W. Grimus and L. Lavoura, The seesaw mechanism at arbitrary order: disentangling the small scale from the large scale, JHEP 11 (2000) 042 hep-ph/0008179.

[10] L. Lavoura, Zeros of the inverted neutrino mass matrix, Phys. Lett. B 609 (2005) 317 hep-ph/0411232.

[11] H. Fritzsch, Weak interaction mixing in the six-quark theory, Phys. Lett. 73B (1978) 317 ;

L.-F. Li, Comments on the derivation of the mixing angles, Phys. Lett. 84B (1979) 461.

[12] S. Kaneko, H. Sawanaka and M. Tanimoto, Hybrid textures of neutrinos, JHEP 08 (2005) 073 hep-ph/0504074.

[13] W. Grimus and L. Lavoura, Soft lepton-flavor violation in a multi-Higgs-doublet seesaw model, Phys. Rev. D 66 (2002) 014016 hep-ph/0204070. 

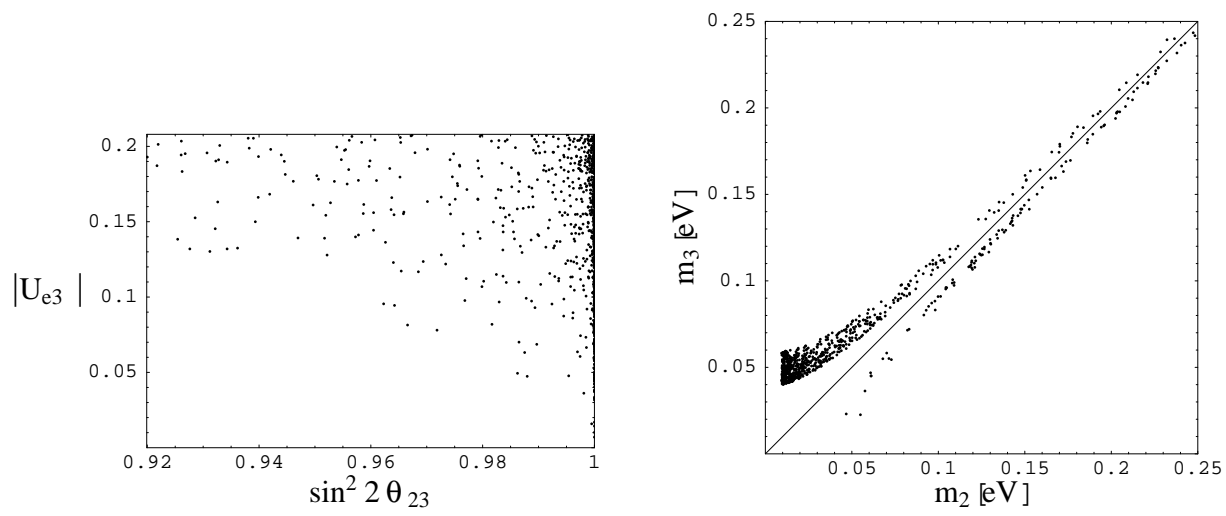

Figure 1: Scatter plots of the allowed region in case 5, where 100,000 random parameter sets are generated.
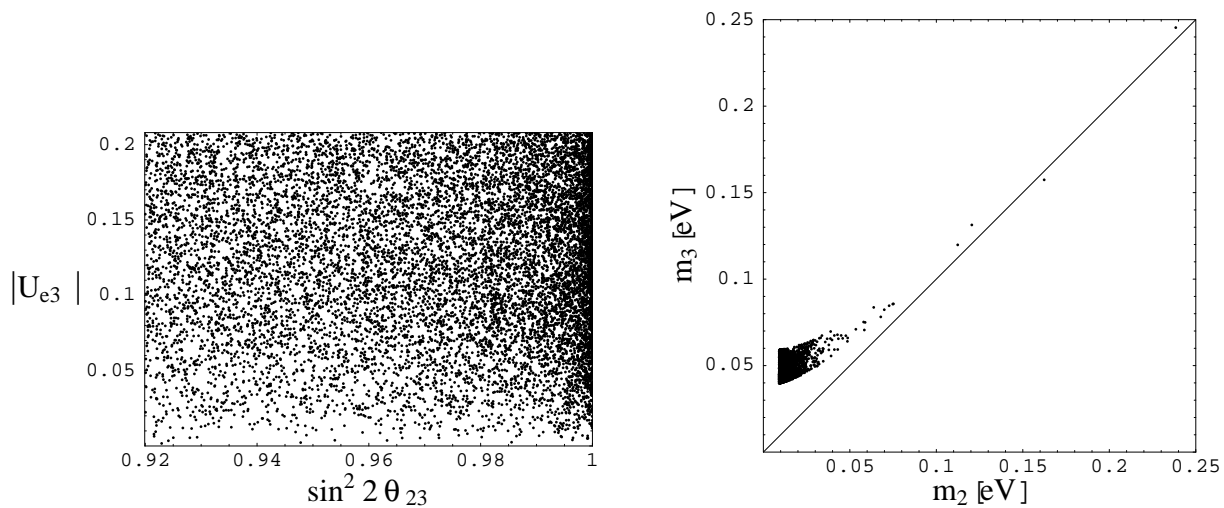

Figure 2: Scatter plots of the allowed region in case 6, where 100,000 random parameter sets are generated.
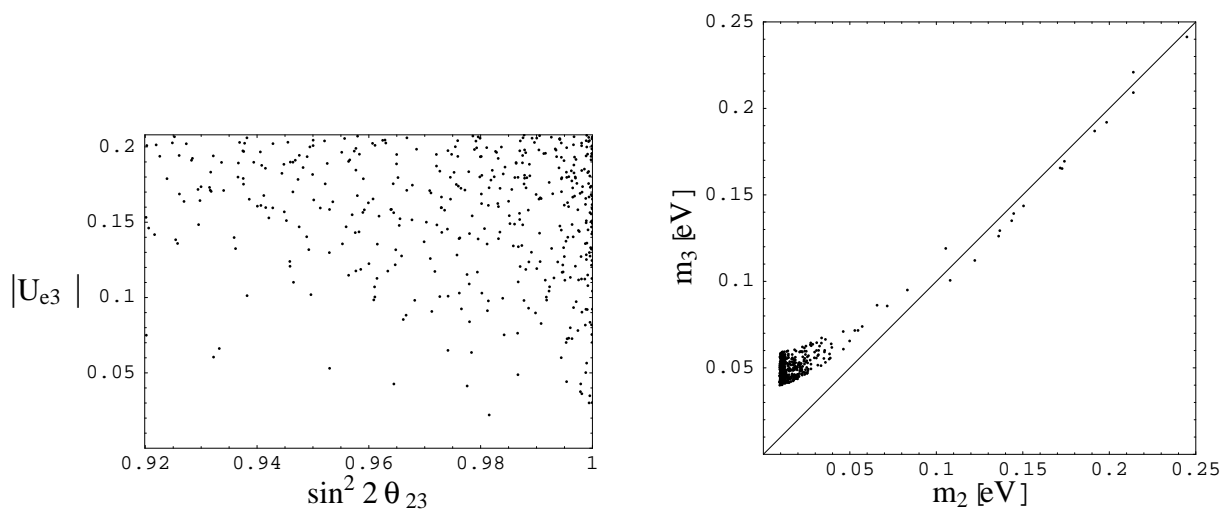

Figure 3: Scatter plots of the allowed region in case 7, where 100,000 random parameter sets are generated. 

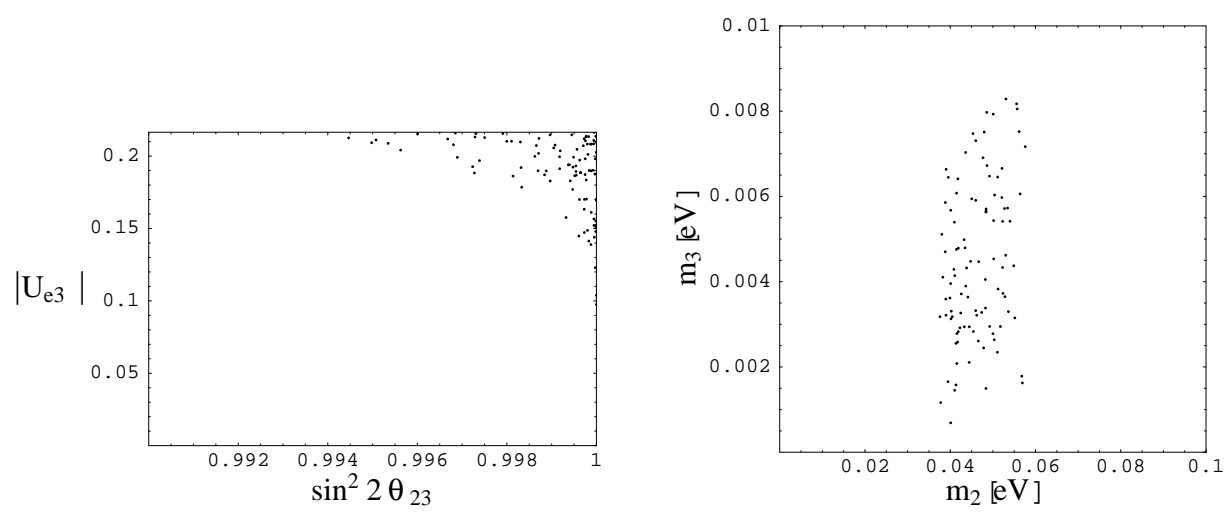

Figure 4: Scatter plots of the allowed region in case 8, where 2,000,000 random parameter sets are generated.
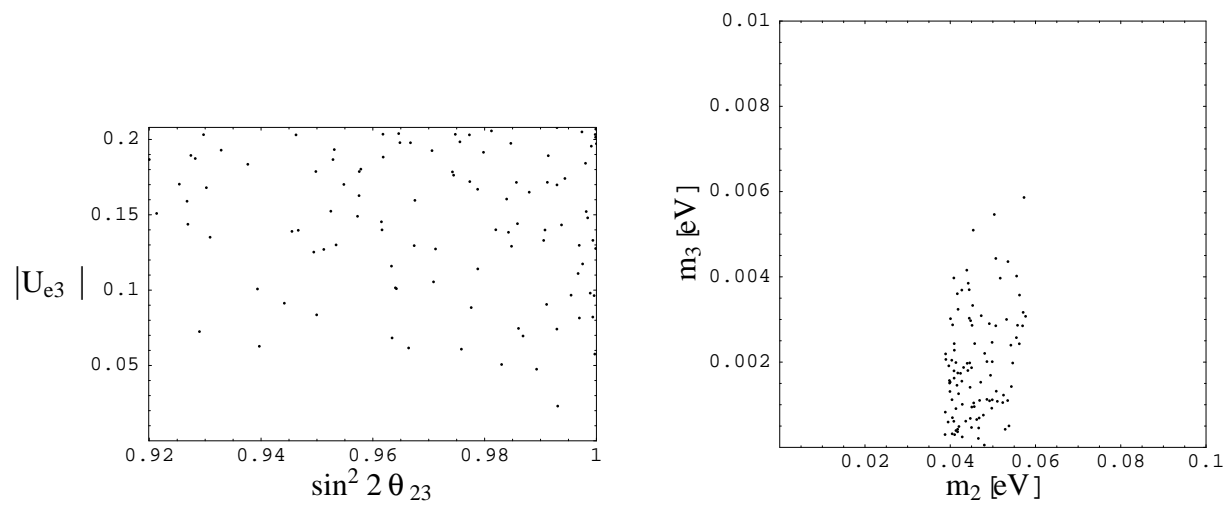

Figure 5: Scatter plots of the allowed region in case 9, where 100,000 random parameter sets are generated.
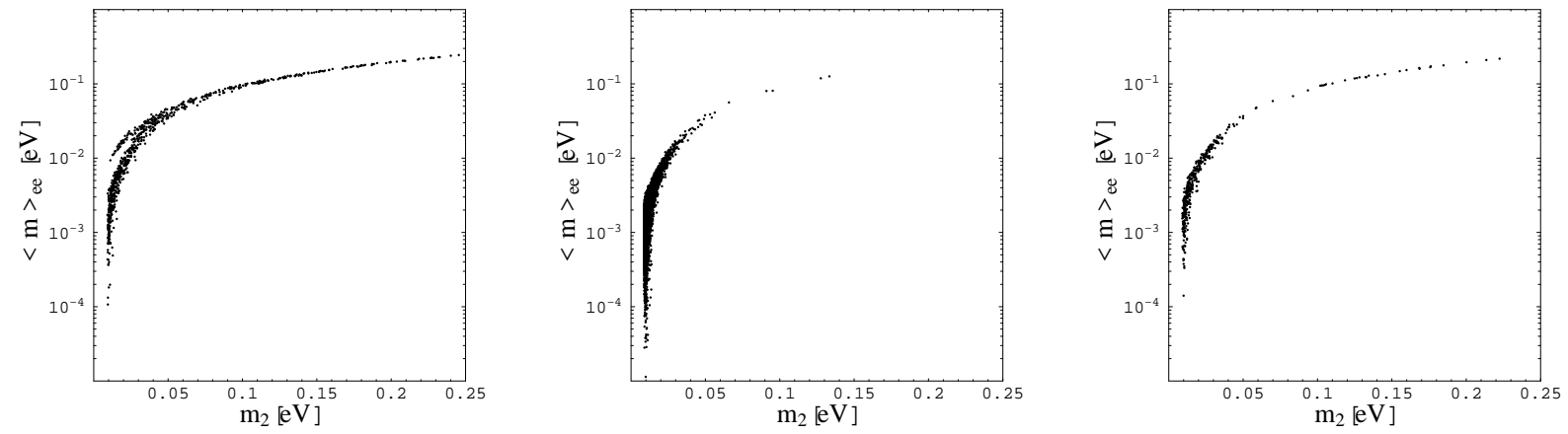

Figure 6: Scatter plots of the $\langle m\rangle_{e e}$ of neutrinoless $\beta \beta$ decay as a function of $m_{2}$ for cases 5,6 , and 7 . 

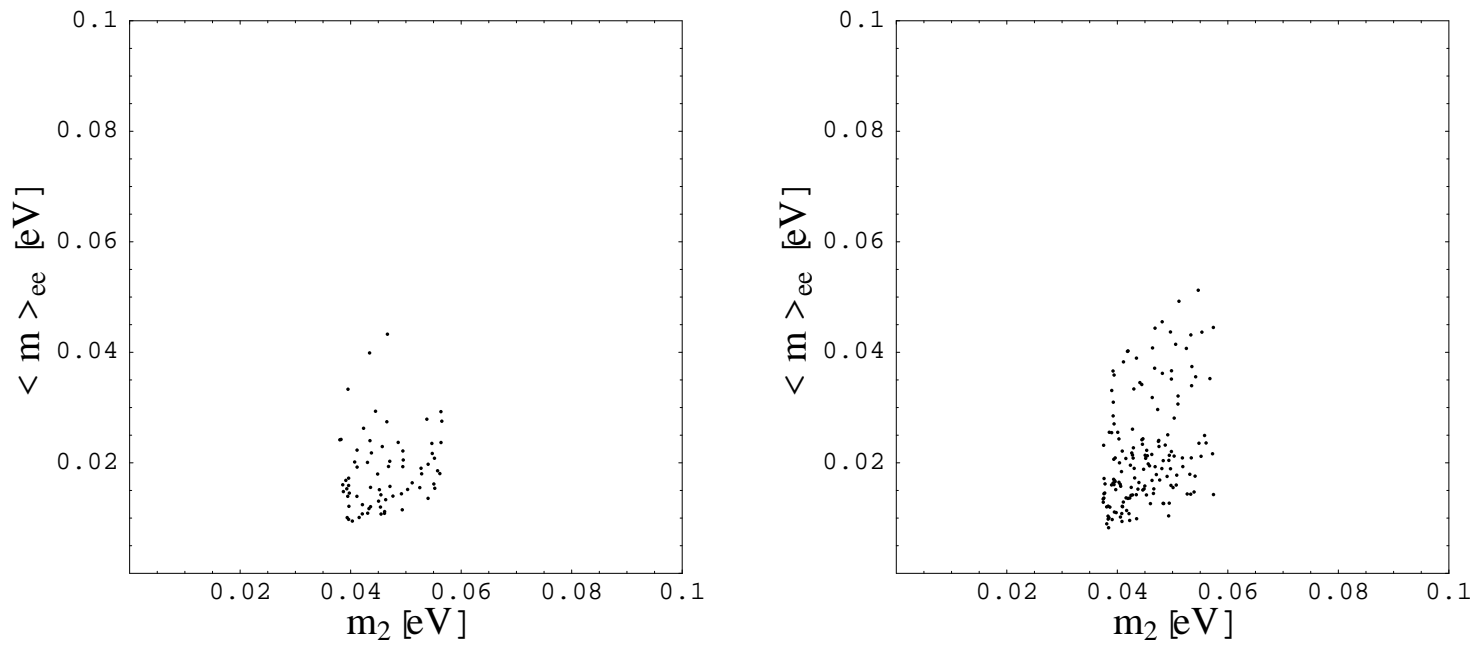

Figure 7: Scatter plots of the $\langle m\rangle_{e e}$ of neutrinoless $\beta \beta$ decay as a function of $m_{2}$ for cases 8 and 9 . 